\title{
A Model for Persistent Levy Motion
}

\author{
A. V. Chechkin and V. Yu. Gonchar \\ Institute for Theoretical Physics \\ National Science Center "Kharkov Institute of Physics and Technology" \\ Akademicheskaya St.1, Kharkov 310108, Ukraine
}

\begin{abstract}
We propose the model, which allows us to approximate fractional Levy noise and fractional Levy motion. Our model is based (i) on the Gnedenko limit theorem for an attraction basin of stable probability law, and (ii) on regarding fractional noise as the result of fractional integration/differentiation of a white Levy noise. We investigate self - affine properties of the approximation and conclude that it is suitable for modeling persistent Levy motion with the Levy index between 1 and 2 .
\end{abstract}

PACS number(s): 02.50.-r, 05.40.+j 


\section{INTRODUCTION.}

By Levy motions, or Levy processes, we designate a class of random functions, which are a natural generalization of the Brownian motion, and whose increments are stationary, statistically self-affine and stably distributed in the sense of P. Levy [1]. Two important subclasses are (i) the stable processes, or the ordinary Levy motions, which generalize the ordinary Brownian motion, or the Wiener process, and whose increments are independent, and (ii) the fractional Levy motions, which generalize the fractional Brownian motions and have an infinite span of interdependence.

The theory of processes with independent increments was developed beginning from the Bachelier's paper [2] concerning Brownian motion. However, the rigorous construction of this process and studies of properties of its trajectories were undertaken by Wiener [3]. The modern presentation of the general theory of processes with independent increments is contained in [4.5]. The theory of stable processes with independent increments has begun its history from the already cited work [1] and, later on, was developed by other prominent mathematicians. In particular, the properties of extrema of stable symmetric processes were studied in Ref. [6]. The local properties of stable processes and geometric properties of their trajectories were considered in Ref. [7].

The basis of the general theory of random processes with stationary self-affine increments was laid by Kolmogorov [8]. Fractional Brownian motions were introduced by Mandelbrot and van Ness as a (relatively) simple family of random functions "that could be in some way be expected to be "typical" of what happens in the absence of asymptotic independence " [9]. In this paper the possibility was also pointed out of constructing "fractional Levy-stable random functions" in a way analogous to that of constructing fractional Brownian motion. Such stable generalizations of fractional Brownian motion were introduced in 10,11].

The Levy random processes play an important role in different areas of application for at least two reasons.

The first one is that the Levy motion can be considered as a generalization of the Brownian motion. Indeed, the mathematical foundation of the generalization are remarkable properties of stable probability laws. From the limit theorems point of view, the stable distributions are a generalization of widely used Gaussian distribution. Namely, stable distributions are the limit ones for the distributions of (properly normalized) sums of independent identically distributed (i.i.d.) random variables [12]. Therefore, these distributions (like the Gaussian one) occur, when the evolution of a physical system or the result of an experiment are determined by the sum of a large number of identical independent random factors. An important distinction of stable probability densities is the power law tails decreasing as $|x|^{-1-\alpha}, \quad x \rightarrow \infty, \alpha$ is the Levy index, $0<\alpha<2$. Hence, the distribution moments of the order $\alpha$ diverge. In particular, stably distributed variables possess a non-finite variance.

The second reason for ubiquity of the Levy motions is their remarkable property of scale invariance. From this point of view the Levy motions (like the Brownian ones) belong to the so - called fractal random processes. Indeed, the objects in nature rarely exhibit exact self similarity ( like the von Koch curve), or self - affinity. On the contrary, these properties have to be understood in a probabilistic sense [13,14. The random fractals are believed to be widely spread in nature. A coastline is a simple example of statistically self - similar object, whereas the trace of the Brownian motion is statistically self - affine. Several numerical 
algorithms were developed in order to simulate fractional Brownian motion [15, 14, 16, 17,. They allow one to model many highly irregular natural objects, which can be viewed as random fractals [14]. The traces of the Levy motions are also statistically self - affine, therefore, one may expect that they are also suited for modeling and studies of natural random fractals.

The stable distributions and the Levy, or Levy - like, random processes are widely used in different areas, where the phenomena possessing scale invariance (in a probabilistic sense) are viewed or, at least, can be suspected, e.g., in economy 18 20, biology and physiology [21], turbulence [22] and chaotic dynamics [23], solid state physics [24], plasma physics [25], geophysics [26] etc. In this respect, the models are needed, which allow one to simulate fractional Levy motion (fLm) with the prescribed quantitative statistical properties and to test and improve methods aimed at analysis and interpretation of experimental data. As far as the authors know, the properties of fLm are, at least, rarely discussed in physics literature. Therefore, we believe, that the models constructed "at the physical level of strictness" may be useful for developing effective methods for experimental data processing and for the purposes of numerical modeling. When studying this problem, we find that the different levels of complexity are required for the models aimed to approximate fLm, which differ by their Levy index $\alpha$ and by their "long-memory" behavior, that is, persistent or anti-persistent one. We discuss this item below and restrict ourselves with the simplest model, which serves as a good approximation to the persistent fLm with the Levy index lying between 1 and 2. We propose a simple approximation to persistent fLm and study its scaling properties.

\section{II. " $\tau^{H}$ LAWS" FOR THE FRACTIONAL LEVY MOTION.}

Our aim is to make an approximation to the process denoted by $L_{\alpha, H}(t)$, whose increments are stably distributed with the Levy index $\alpha, 0<\alpha \leq 2$, and possess the properties of stationarity and self - affinity. By analogy with the Definition 3.2 of Ref. [9], the increments of a random function $L_{\alpha, H}(t)$ will be said to be self - affine with parameter $H$ if for any $\kappa>0$ and any $t_{0}$

$$
L_{\alpha, H}\left(t_{0}+\kappa \tau\right)-L_{\alpha, H}\left(t_{0}\right) \stackrel{d}{=}\left[\kappa^{H}\left(L_{\alpha, H}\left(t_{0}+\tau\right)-L_{\alpha, H}\left(t_{0}\right)\right)\right]
$$

The particular cases are:

(i) ordinary Brownian motion, $L_{\alpha, H}(t) \equiv B(t)$, which has $H=1 / 2$;

(ii) fractional Brownian motion, $L_{\alpha, H}(t) \equiv B_{H}(t)$, which has $0<H<1$;

(iii) ordinary Levy motion, $L_{\alpha, H}(t) \equiv L_{\alpha}(t)$, which has $H=1 / \alpha$.

Since the Levy-stable distribution with the Levy index $\alpha$ possesses the moments of the order $q<\alpha$, the " $\tau^{H}$ laws" (by terminology of [9]) for the structure functions of the fractional Levy motion can be stated as follows: for any $0<q<\alpha$

$$
\left\langle\left|L_{\alpha, H}(t+\tau)-L_{\alpha, H}(t)\right|^{q}\right\rangle^{1 / q} \propto \tau^{H}
$$

where the proportionality coefficient is a function of $q$ and the parameters of stable distribution for the increments, but not of $H$. The range of $H$ can be determined as follows. By Minkowski's inequality, for any positive $\tau_{1}, \tau_{2}>0$ and $1<q<\alpha$ 


$$
\begin{gathered}
\left\langle\left|L_{\alpha, H}\left(t+\tau_{1}+\tau_{2}\right)-L_{\alpha, H}(t)\right|^{q}\right\rangle^{1 / q} \leq \\
\left\langle\left|L_{\alpha, H}\left(t+\tau_{1}+\tau_{2}\right)-L_{\alpha, H}\left(t+\tau_{1}\right)\right|^{q}\right\rangle^{1 / q}+ \\
+\left\langle\left|L_{\alpha, H}\left(t+\tau_{1}\right)-L_{\alpha, H}(t)\right|^{q}\right\rangle^{1 / q}
\end{gathered}
$$

or, using Eq.(2),

$$
\left(\tau_{1}+\tau_{2}\right)^{H} \leq \tau_{1}^{H}+\tau_{2}^{H}
$$

which implies $H \leq 1$. Further, we require $q$ - th order mean continuity of $L_{\alpha, H}(t)$, that is [甘], for any $t$ and $q<\alpha$

$$
\lim _{\Delta t \rightarrow 0}\left\langle\left|L_{\alpha, H}(t+\Delta t)-L_{\alpha, H}(t)\right|^{q}\right\rangle=0
$$

It implies that $H \geq 0$.

From Eq. (1) the " $\tau^{H}$ law" follows also for the range of the fLm with the Levy index $\alpha>1$,

$$
\begin{gathered}
R(\tau)=\sup _{0 \leq s \leq \tau}\left(L_{\alpha, H}(s)-L_{\alpha, H}(0)\right)-\inf _{0 \leq s \leq \tau}\left(L_{\alpha, H}(s)-L_{\alpha, H}(0)\right) \\
\langle R(\tau)\rangle \propto \tau^{H}
\end{gathered}
$$

We make some remarks concerning distinction between the motions with the Levy index lying between 0 and 1, and those with the index lying between 1 and 2 . Comparatively to the latter case, the former one is more involved for the analysis and numerical simulation. The reasons are as follows:

(i) since $q$ must be less than $\alpha$, the Minkowski's inequality does not hold for $\alpha \leq 1$, hence we can not use it for establishing the upper boundary for $H$;

(ii) the statistical mean of the range is infinite for $\alpha \leq 1$;

(iii) with $\alpha$ decreasing, the increments of fLm grow rapidly due to the power - law tails of stable distributions. Very large increments are occurring more and more frequently, and the moments of the increments strongly fluctuate from realization to realization. Thus, the reliability of the results of simulation decreases. The same conclusion can be done when treating experimental data, in which the Levy statistics with $\alpha<1$ can, at least, be suspected.

The three peculiarities mentioned above require a modification of the methods, which we use below for making an approximation to fLm and for studying its properties. For this reason, we restrict ourselves by the case $1 \leq \alpha \leq 2$, however, having in mind to study more complicated case $0<\alpha<1$ in future. Fortunately for us, there are evidences from different areas of application, see, e.g., [18] [26], that, as a rule, the Levy processes with $\alpha>1$ do occur. 


\section{THE MODEL.}

The process of constructing approximation to fLm can be divided into 3 steps.

Step 1. At the first step we generate random sequence of i.i.d. random variables possessing stable probability law. These variables play the role of increments of the ordinary Levy motion having the Levy index $\alpha, 0<\alpha<2$. The value $\alpha=2$ corresponds to the ordinary Brownian motion, hence in this case the sequence of independent increments is generated with the use of a standard Gaussian generator. Since in [15] the sequence generated at $\alpha=2$ is called "approximate discrete-time white Gaussian noise" one may call the sequence generated at $0<\alpha<2$ "approximate discrete-time white Levy noise".

We restrict ourselves by symmetric stable laws with the probability density $p_{\alpha, D}(x)$ and the characteristic function

$$
p_{\alpha, D}(k)=\langle\exp (i k x)\rangle=\exp \left(-D|k|^{\alpha}\right)
$$

Here $\alpha$ is the Levy index, and $D$ is a positive parameter. At $\alpha=1$ and 2 one has the Cauchy and the Gaussian probability laws, respectively. In other cases the symmetric stable laws are not expressed in terms of elementary functions. At $0<\alpha<2$ they have power law asymptotic tails [12],

$$
p_{\alpha, D}(x) \propto D \frac{\Gamma(1+\alpha) \sin (\pi \alpha / 2)}{\pi|x|^{1+\alpha}}, \quad x \rightarrow \pm \infty .
$$

Among the methods of random sequence generation with the given probability law $F(x)$ the method of inversion seems most simple and effective [27]. However, it is well-known that its validity is limited by the laws possessing analytic expressions for $F^{-1}$, hence, the direct application of the method of inversion to the stable law is not expedient. In this connection, we exploit an important property of stable distributions. Namely, such distributions are limiting for those of properly normalized sums of i.i.d. random variables [12]. To be more concrete, we generate the needed random sequence in two steps. At the first one we generate an "auxiliary" sequence of i.i.d. random variables $\left\{\xi_{j}\right\}$, whose distribution density $F^{\prime}(x)$ possesses asymptotics having the same power law dependence as the stable density with the Levy index $\alpha$ has, see Eq.(9). However, contrary to the stable law, the function $F(x)$ is chosen as simple as possible in order to get analytic form of $F^{-1}$. For example,

$$
\begin{aligned}
& F(x)=\frac{1}{2\left(1+|x|^{\alpha}\right)}, \quad x<0, \\
& F(x)=1-\frac{1}{2\left(1+x^{\alpha}\right)} \quad x>0 .
\end{aligned}
$$

Then, the normalized sum is estimated,

$$
X=\frac{1}{a m^{1 / \alpha}} \sum_{j=1}^{m} \xi_{j}
$$

where 


$$
a=\left(\frac{\pi}{2 \Gamma(\alpha) \sin (\pi \alpha / 2)}\right)^{1 / \alpha}
$$

According to the Gnedenko theorem on the normal attraction basin to the stable law [12], the distribution of the sum (10) converges to the stable law with the characteristic function (8) and $D=1$. It is reasonable to generate the stable sequences with $D=1$, doing the rescaling after that, if necessary. Repeating $N$ times the above procedure, we get a sequence of i.i.d. random variables $X(t), t=1,2, \ldots, N$. This is an approximation to a discrete - time white Levy noise.

Step 2. At the second step we convert approximate white Levy noise $X(t)$ into the approximate fractional Levy noise $X_{\nu}(t)$. For this purpose one can use the procedure, which is called fractional integration/differentiation of a white noise [15]. We remind the relation between the Fourier transforms of the function $X(t)$ and of its fractional integral/derivative $X_{\nu}(t)$ of the $\nu$-th order:

$$
\stackrel{\wedge}{X}_{\nu}(\omega)=\frac{\hat{X}(\omega)}{(-i \omega)^{\nu}}
$$

where $\nu$ is positive in case of fractional integration and negative in case of fractional differentiation (to be more accurate, we say about left-side Riemann - Liouville fractional integral/derivative at the infinite axis [28]). Fractional integration of a white noise leads to amplification of low-frequency spectral components of the noise and thus, to the persistent process, whereas fractional differentiation leads to amplification of high-frequency spectral components and thus, to the anti-persistent process. At a qualitative level the difference between the persistent and anti-persistent behaviors can be formulated as follows: in the persistent random process the available tendency is supported, whereas in the anti-persistent process the opposite tendency prevails [15] [29]. The range of $\nu$ will be discussed below. The approximate discrete - time fractional Levy noise $X_{\nu}(t)$ plays the role of the sequence of increments of the approximation to fLm.

Step 3. With using approximate discrete - time fractional Levy noise $X_{\nu}(t)$ the approximation to the fractional Levy motion is defined by

$$
L_{\alpha, \nu}(t)=\sum_{\tau=1}^{t} X_{\nu}(\tau)
$$

Let us discuss the restrictions on the possible values of $\nu$. They are dictated by the restrictions on the range of $H$ for the fLm, which we intend to approximate.

At first, we note that the approximation to the white Levy noise possesses the property of self - affinity,

$$
X(\kappa t) \stackrel{d}{=} \kappa^{1 / \alpha} X(t)
$$

Here $\kappa$ is a positive integer. It follows from Eq. (13) that

$$
\stackrel{\wedge}{X}\left(\frac{\omega}{\kappa}\right) \stackrel{d}{=} \kappa^{1+1 / \alpha} \stackrel{\wedge}{X}(\omega)
$$


and thus, according to our way of modeling,

$$
X_{\nu}(\kappa t) \stackrel{d}{=} \kappa^{\nu+1 / \alpha} X_{\nu}(t)
$$

This is the property of self - affinity of the increments of the approximation to fLm. By comparing it with Eq.(1), we conclude that the equality

$$
H=\nu+1 / \alpha
$$

must hold. Taking into account the restrictions on $H$, we conclude that for $1 \leq \alpha \leq 2$

$$
-1 / \alpha \leq \nu \leq 1-1 / \alpha
$$

The particular cases of the proposed approximation are as follows:

(i) $\alpha=2, \nu=0$. In this case we get approximation to the Gaussian process with independent increments, that is, to the ordinary Brownian motion, which has the parameter $H$ equal $1 / 2$;

(ii) $\alpha=2,-1 / 2<\nu<1 / 2$. In this case we get approximation to the fractional Brownian motion, which has the parameter $H$ lying between 0 and 1 . We studied the properties of this approximation in [30];

(iii) $0<\alpha<2, \quad \nu=0$. In this case we get approximation to the ordinary Levy motion, which has the parameter $H$ equal $1 / \alpha$. The step 2 is omitted when getting this approximation. Its properties were studied in [31. The three different models of the ordinary Levy motions were proposed in [32]. They can be interpreted as a "difference scheme" to approximate the evolution equation for the density distribution of the ordinary Levy motion.

In Fig.1 the admissible range of the index $\nu$ is shown for $1<\alpha \leq 2$ on the $(\alpha, \nu)$ plane. This range is bounded by the curve $H=\nu+1 / \alpha=1$ in the top and by the curve $H=0$ in the bottom. The right vertical boundary indicated by thick line corresponds to the fractional Brownian motion, $\alpha=2,-1 / 2<\nu<1 / 2$. The horizontal thick line $\nu=0$ corresponds to the ordinary Levy motion. This line divides two regions: upper one, $\nu>0$, in which the Levy motion is persistent, and the bottom one, $\nu<0$, in which the motion is anti-persistent. Dotted lines $a, b$ relate to Figs. 4, 5 and will be explained below.

Before proceeding with the numerical results illustrating the self-affinity properties of our approximation, we discuss the peculiarities of simulating persistent and anti-persistent motions. The problem appearing can be explained by taking fractional Brownian motion as an example [30]. At first we consider the persistent case. Fractional integration of a white Gaussian noise leads to the fractional Gaussian noise with spectral density decreasing as frequency increases. Thus, at numerical simulation the high-frequency inaccuracies are small and the low-frequency ones play the main role. Now we turn to the anti-persistent case. Fractional differentiation of a white Gaussian noise leads to the fractional Gaussian noise with spectral density increasing as frequency increases. At numerical simulation the high-frequency inaccuracies are prevailing. We have demonstrated, when studying fractional Brownian motion approximation based on fractional integration/differentiation of a white noise [30], that the high - frequency inaccuracies are more essential than the low - frequency ones, that is, the anti - persistent case is modeled with less accuracy. Moreover, it is known that the other algorithms for simulating fractional Brownian motion, namely those proposed 
in [15] and in [14] as well, have the same drawback. Thus, one may expect that the antipersistent fLm is also worse modeled. This is indeed so, as we convinced during numerical simulation. When simulating fractional Brownian motion, the problem is overcome in part by passing from "Type -2 Approximation" to a more sophisticated "Type 1 Approximation" having a finer grid [15]. A similar improvement can be performed when doing fractional differentiation of a white Levy noise. However, such a procedure requires more analysis, which have to be the subject of a separate paper. That is why below we present the results for the persistent case only. We also note that the persistent behavior is prevalent in nature [15] [29], thus, we may hope that approximation to the persistent fractional Levy motion is more needed for applications. Of course, this does not imply that there is no need for studying anti - persistent fLm. On the contrary, we have in mind that the complex systems with the feedback require the development of approximations well-suited for modeling anti - persistent behavior. As an example we quote long - range anti - correlations and non Gaussian behavior of the heartbeat of the healthy subjects [33.

\section{NUMERICAL RESULTS.}

The results of numerical simulation and analysis are shown in Figs. 2 - 5 .

In the top of Fig. 2 the probability densities $p(x)$ for the members of the sequence $X(t)$ are depicted by black points for (a) $\alpha=1.2$, and (b) $\alpha=1.7$. We use $m=30$ terms in the sum (10). The functions $p_{\alpha, 1}(x)$ obtained by the inverse Fourier transform, see Eq.(8), are shown by solid lines. In the bottom of Fig. 2 the black points depict asymptotics of the same probability densities in log - log scale. The solid lines show the asymptotics given by Eq. (9). It is seen that the Levy index can be estimated with the use of those values of $X(t)$, which lie outside the peak located around $x=0$. For the comparison the asymptotics of the Gaussian distribution with zero mean and unit variance are also shown. The log - log scale allows one to demonstrate smallness of probability of extreme Gaussian events. The examples presented demonstrate a good agreement between the probability densities for the sequences $X(t)$ obtained with the use of the numerical algorithm proposed and the densities of the stable laws.

We would like to note that the simplicity is a certain merit of the proposed approximation to a white Levy noise. The approximation is entirely based on classical formulation of one of the limit theorems and can be easily generalized for the case of asymmetric stable distributions. It also allows one, after some modifications, to speed up the convergence to the stable law. These problems, however, ought to be the subject of a separate paper. We also note, that two schemes were proposed recently, which use the combinations of random number generators [34] and the family of chaotic dynamical systems with broad probability distributions [35], respectively.

In Fig. 3 typical samples of approximation to discrete - time Levy noises with the Levy index $\alpha=1.5$ are depicted for a white noise, $\nu=0$ (at the left), and for a fractional one, $\nu=0.3$ (at the right). In both cases large frequent "outliers" are clearly seen, which are totally absent in case of the Gaussian noises. An interesting feature of the top figures is that they look very similar. Indeed, only careful comparison can reveal the differences between them. However, the corresponding ordinary Levy motion shown below at the left, and fractional persistent Levy motion shown below at the right are strikingly different. It 
is so, because the fractional noise possesses such correlations, that the values of the noise at different instants "acts coherently". It means that in case of persistent motion, not only "jumps", or "Levy flights" (which are clearly visible on both trajectories) lead to a large departure of the trajectory from the $x$ - axis, but also "coordinated action" of the noise at different instants results in such a departure. In terms of anomalous diffusion we may argue that, contrary to the case of the ordinary Levy motion, when an anomalous diffusion rate is determined almost solely by the "Levy flights", in the persistent Levy motion it is also determined by "small steps". This is a qualitative corollary of the procedure of fractional integration of a white noise.

In Fig. 4 we illustrate the properties of structure functions. We study $\tau$ - dependence of the structure functions of our approximation,

$$
\left\langle\left|L_{\alpha, \nu}(t+\tau)-L_{\alpha, \nu}(t)\right|^{q}\right\rangle^{1 / q} \propto \tau^{s},
$$

where $q<\alpha$. Since for the fLm the " $\tau^{H}$ law" is fulfilled, see Eq.(1), it is expected, according to our way of modeling, that $s$ is close to $\nu+1 / \alpha$. We demonstrate the results for $q=1 / 4$. However, we verified that for any $q$ less than $\alpha$ and not very close to it the results are just the same. We present the results for the approximation to the persistent Levy motions, whose parameters $\alpha, \nu$ vary along dotted lines $a, b$ shown in Fig. 1.

In Fig. 4a the results for the vertical line $a$ are presented, $\alpha=1.7$. The exponent $s$ versus $\nu$, see Eq.(18), is depicted by black squares. The expected relation $s=\nu+1 / \alpha$ is shown by solid line. We see that numerical results are well fitted by the expected line, but the discrepancy appears when $\nu$ reaches its upper boundary, that is, for the strongly persistent case. The same effect appears when simulating fractional Brownian motion, $\alpha=2$ [30]. Since the Fourier transform of a white noise is divided by $\omega^{\nu}, \nu>0$, when getting fractional noise in the persistent case (see Step 2 of the approximation), one may suppose that the appeared discrepancy is due to the growth of low - frequency inaccuracies with $\nu$ increasing. However, this is not a single reason: indeed, we convinced that making longer sample path does not diminish discrepancy essentially.

In Fig. $4 \mathrm{~b}$ the results for the processes along the dotted curve $b$ (see Fig.1) are presented. The curve $b$ corresponds to the case $H=\nu+1 / \alpha=0.8$. In the figure the $y$-axis indicates the values of $s$, whereas along the $x$-axis both the values of $\alpha$ (in the bottom, linear scale) and of $\nu$ (in the top, non - linear scale) are shown. Black squares indicate the values of $s$ measured along the curve $b$, whereas solid line indicates the value $s=0.8$, which we expect to get. We see, that numerical results are well fitted by the expected line. For the comparison we show by crosses the exponent $s$ measured from Eq. (18) for the second order structure function, $q=2$. The theoretical second order structure function is infinite for the Levy motion. This circumstance has two consequences for the numerical simulation and/ or at experimental data processing. The first one is that the second order structure function increases with the length of the Levy motion trajectory increasing. The second consequence is that the exponent $s$ does not depend on $\alpha$ and demonstrates "pseudo - Gaussian" behavior. Indeed, the dotted line $s=\nu+1 / 2$ (which indicates the relation between $s$ and $\nu$ for the approximation to the fractional Brownian motion) well fits the relation indicated by crosses in Fig. $4 b$.

In Fig.5 we illustrate the studies of the range. We investigate $\tau$ - dependence of the range $R_{\nu}$ of our approximation to the fLm with the Levy index $\alpha>1$, 


$$
\begin{gathered}
R_{\nu}(\tau)=\sup _{0 \leq s \leq \tau}\left[L_{\alpha, \nu}(s)-L_{\alpha, \nu}(0)\right]-\inf _{0 \leq s \leq \tau}\left[L_{\alpha, \nu}(s)-L_{\alpha, \nu}(0)\right], \\
\left\langle R_{\nu}(\tau)\right\rangle \propto \tau^{H_{\nu}} .
\end{gathered}
$$

Since the " $\tau^{H}$ law" is fulfilled for the range of the Levy motion, see Eqs. $(6,7)$, it is expected, according our way of modeling, that $H_{\nu}$ is close to $H=\nu+1 / \alpha$.

In the empirical rescaled range analysis, that is, at experimental data processing, or in numerical simulation, the range of the random process is divided by the standard deviation of its increments after subtraction of a linear trend [36]. This procedure, called the Hurst method, or the method of normalized range, in particular, smooths the variations of the range on different segments of time series. As the result of the empirical rescaled range analysis of experimental data, one gets the Hurst exponent of the process, that is, the exponent $H_{\nu}$ in our case. However, the Hurst method is not satisfactory for the Levy motion because of the infinity of the theoretical value of the standard deviation. Therefore, we propose to modify the Hurst method by exploiting the $1 / \alpha$ - th root of the $\alpha$ - th moment instead of standard deviation, that is,

$$
\sigma_{\alpha}=\left(\frac{1}{\tau} \sum_{t=1}^{\tau}|X(t)|^{\alpha}\right)^{1 / \alpha}
$$

Since it has only weak logarithmic divergence with the number of terms in the sum increasing, then the power - law dependence (20) is not changed.

In Fig. 5a we present the results of applying the modified Hurst method to the ranges of the processes with the parameters along the vertical line $a$ in Fig. 1. The Levy index $\alpha$ is equal 1.7. In the figure the exponent $H_{\nu}$ versus $\nu$ is depicted by black squares. The relation $H_{\nu}=\nu+1 / \alpha$ is shown by solid line. As in case of the structure function, see Fig. 4a, we notice that numerical results are well fitted by the line showing the expected relation. However, the discrepancy appear for $\nu$ reaching its upper boundary, that is, for the strongly persistent case. In this respect we may repeat our above comment to the analogous discrepancies in case of the structure function.

In Fig. 5b we present the results of applying the modified Hurst method to the ranges of the process with the parameters changing along the curve $b$ in Fig. 1. This curve corresponds to the case $H=\nu+1 / \alpha=0.8$. The $y$ - axis indicates the values of $H_{\nu}$, whereas along the $x$ axis both the values of $\alpha$ (in the bottom, linear scale) and of $\nu$ (in the top, non - linear scale) are shown. Black squares indicate the values of $H_{\nu}$ measured along the curve $b$, whereas solid line indicates the value $H_{\nu}=0.8$, which we expect to get. We see the discrepancy between the numerical results and the expected line. However, the discrepancy is even larger, if one uses the ordinary, or non - modified, Hurst method. The results of its exploiting are shown by crosses, whereas the "pseudo - Gaussian" relation $H_{\nu}=\nu+1 / 2$ (which does not depend on $\alpha$ ) is depicted by dotted line. One can clearly see the inapplicability of the "traditional" Hurst method for characterizing properties of the Levy motions.

\section{RESULTS}

The results of the paper are as follows. 
1. We propose a model for the random process, whose increments are stationary, self - affine and distributed with the stable probability law. By analogy with the fractional Brownian motion, the family of these processes can be called fractional Levy motion.

2. When constructing our model, the two basic steps are:

(i) the use of the Gnedenko limit theorem for the normal attraction basin of a stable law; the theorem gives us a simple way for generating a sequence of independent stably distributed variables, which approximate a discrete - time white Levy noise;

(ii) fractional integration/differentiation of a white noise; this procedure converts white noise into a fractional one, thus allowing us to approximate a discrete - time fractional Levy noise.

3. We find the ranges of the change of the order of fractional integration/differentiation, inside of which the increments of the Levy motion approximation obtained possess the property of self - affinity. This property manifests itself in so - called " $\tau^{H}$ laws", that is, in the power - law time - dependence of both the structure function of the order $q$ less than the Levy index $\alpha$ and the range. The relation between the exponent $H$ and the order of fractional integration/differentiation is also obtained.

4. We study the " $\tau^{H}$ law" for the structure functions of the approximation and find a good agreement between numerical results and the theory for the $q$ - th order structure function, $q<\alpha$. We also demonstrate that the second order structure function has a "pseudo - Gaussian" time behavior, which is irrespective of the Levy index $\alpha$.

5. We study the " $\tau^{H}$ law" for the range and demonstrate, that the "traditional" Hurst method exploiting the normalized rescaled range leads to a "pseudo - Gaussian" time dependence, which is irrespective of the Levy index $\alpha$. Thus, we propose the modified Hurst method, in which the $1 / \alpha$ - th root of the $\alpha$ - th moment of the sequence of increments is used instead of standard deviation. The modified Hurst method leads to the rescaled range time - dependence, which is much closer to the theory than that of normalized range.

6. We conclude that our approximation is suitable for simulating persistent Levy motion with the Levy indexes varying between 1 and 2. We also discuss the possible reasons why our model works worse in the anti - persistent region and in the region of Levy indexes less than 1 .

7. As it concerns with the "pseudo - Gaussian" effects described above, they allow us to suggest that at estimating the second order structure function and normalized span from experimental data the "Levy nature " of them can be easily masked. This, in turn, rises an interesting task of developing statistical methods for extracting reliable characteristics from experimental data, for which Levy statistics can be expected from, e.g., some physical reasons.

\section{ACKNOWLEDGMENTS}

This work was supported in finance by National Academy of Science of Ukraine, the Project "Chaos-2" and by INTAS Program, the Projects 93-1194, LA-96-09 and 98 - 01. 


\section{REFERENCES}

[1] P. Levy, Theorie de l'Addition des Variables Aleatories (Gauthier - Villiers, Paris, 1937).

[2] L. Bachelier, Annales Scientifiques de l'Ecole Normale Superieure III 17 (1900) 21.

[3] N. Wiener, Journ. Math. Phys. Mass. Inst. Technology 2 (1923) 131.

[4] A. V. Skorokhod, Random Processes with Independent Increments (in Russian: Nauka, Moscow, 1964. Engl. transl.: Kluwer, Dordrecht, 1991);

[5] G. Samorodnitsky, M. S. Taqqu, Stable non - Gaussian Random Processes (Chapman \& Hall, New York, 1994).

[6] D. A. Darling, Trans. Amer. Math. Soc. 83 (1956) 164.

[7] R. M. Blummental, R. K. Getoor, Trans. Amer. Math. Soc. 95 (1960) 263; Journ. Math. 4 (1960) 370.

[8] A. N. Kolmogorov, C.R. (Doklady) Acad. Sci. URSS 26 (1940) 115.

[9] B. B. Mandelbrot and van Ness, SIAM Review 10 (1968) 422.

[10] M. S. Taqqu, R. Wolpert, Z. Wahrsch. Verw. Gebiete 62 (1983) 53.

[11] M. Maejima, Z. Wahrsch. Verw. Gebiete 62 (1983) 235.

[12] B. V. Gnedenko, A. N. Kolmogorov, Limit distributions for Sums of Independent Random Variables (in Russian: Izd-vo tekhniko-teor. lit-ry , Moskva, 1949; Engl. transl.:Addison Wesley, Reading, MA, 1954).

[13] B. B. Mandelbrot, The Fractal Geometry of Nature (Freeman, New York, 1982).

[14] R. F. Voss, in: Fundamental Algorithms in Computer Graphics, edited by R. A. Earnshaw (Springer-Verlag, Berlin, 1985), p.805.

[15] B. B. Mandelbrot and J. R. Wallis, Water Resources Research 5 (1969) 228.

[16] S. Rambaldi, O. Pinazza, Physica A 208 (1994) 21.

[17] O. Magre, M. Guglielmi, Chaos, Solitons and Fractals 8 (1997) 377.

[18] B. B. Mandelbrot, Journ. Business 36 (1963) 394;

B. B. Mandelbrot, Fractals and Scaling in Finance (Springer-Verlag, New York, 1997).

[19] R. N. Mantegna, Physica A 179 (1991) 232.

[20] R. N. Mantegna, H. E. Stanley, Physica A 254 (1998) 77.

[21] B. J. West and W. Deering, Phys. Reports 246 (1994) 1.

[22] M. F. Schlesinger, B. J. West, J. Klafter, Phys. Rev. Lett. 58 (1987) 1100.

[23] M. F. Schlesinger, G. M. Zaslavsky, J. Klafter, Nature 363 (1993) 31;

G. M. Zaslavsky, Physica D 76 (1994) 110 ; M. F. Schlesinger (editor), G. M. Zaslavsky, U. Frisch, Levy Flights and Related Topics in Physics: Proceedings of the International Workshop Held at Nice, France, 27 - 30 June 1994 (Springer, Berlin, 1995).

[24] J. - P. Bouchaud, A. Georges, Phys. Reports 195 (1990) 127.

[25] G. Zimbardo, P. Veltri, G. Basile, S. Principato, Phys. Plasmas 2 (1995) 2653.

[26] D. Schertzer, S. Lovejoy, Multifractals and Turbulence: fundamentals and applications (World Scientific, Singapore, 1993).

[27] M. G. Kendall, S. B. Babington, Random Sampling Numbers (Cambridge Univ. Press, Cambridge, 1939).

[28] S. G. Samko, A. A. Kilbas and O. I. Marichev, Fractional Integrals and Derivatives, Theory and Applications (in Russian: Nauka i Tekhnika, Minsk, 1987; Engl. transl.: Amsterdam, Gordon and Breach, 1993).

[29] J. Feder, Fractals (Plenum Press, New York, 1988).

[30] V. Chechkin, V. Yu. Gonchar, Preprint cond - mat/9902209. 
[31] A. V. Chechkin, V. Yu. Gonchar, Preprint cond - mat/9901064.

[32] F. Mainardi, P. Paradisi and R. Gorenflo, accepted to Physica A.

[33] C. - K. Peng, J. Mietus, J. M. Hausdorff, S. Havlin, H. E. Stanley, A. L. Goldberger, Phys. Rev. Lett. 70 (1993) 1343.

[34] R. N. Mantegna, Phys. Rev. E 5B (1994) 4677.

[35] K. Umeno, Phys. Rev. E58 2644 (1998).

[36] H. E. Hurst, R. P. Black, and Y.M. Sinaika, Long Term Storage in Reservoirs. An Experimental Study (Constable, London, 1965). 
Fig. 1. Shaded region indicates the admissible range of the order $\nu$ of fractional integration/differentiation of a white Levy noise. The Levy index $\alpha$ is varied from 1 to 2 . The vertical thick line at the right corresponds to the fractional Brownian motion, $\alpha=2$, $-1 / 2<\nu<1 / 2$. The horizontal thick line $\nu=0$ corresponds to the ordinary Levy motion. Above the horizontal line the process is persistent, whereas below it is anti - persistent. Along the vertical dotted line $a$ the Levy index $\alpha$ is 1.7. Along the dotted line $b$ the relation $\nu+1 / \alpha=0.8$ holds. The self - similarity properties of the approximation to fLm along the dotted lines are illustrated in Figs. 4, 5.

Fig. 2. Probability densities (above) and their asymptotics (below) are shown for the approximation to discrete - time white Levy noises with the Levy indexes $\alpha=1.2$ and $\alpha=1.7$. The probability densities and the asymptotics of the stable laws are indicated by solid lines. Below for the qualitative comparison the asymptotics of the Gaussian distribution are shown.

Fig. 3. Above: typical samples of approximation to a discrete - time white Levy noise, $\nu=0$ (at the left) and fractional Levy noise, $\nu=0.3$ (at the right). Below: corresponding trajectories of approximation to the ordinary Levy motion (at the left) and the fractional Levy motion (at the right).

Fig. 4. (a) Exponent $s$ of the structure function of order $q=1 / 4$ versus the order of fractional integration $\nu$, see Eq.(18) (black points). Processes along the vertical dotted line $a$ from Fig.1 are investigated. The Levy index $\alpha$ is 1.7. Solid line indicates the relation $s=\nu+1 / \alpha$. (b) Exponent $s$ is indicated for the processes along the dotted line $b$ from Fig. 1 (black points). The parameters $\alpha, \nu$ of these processes obey the law $\nu+1 / \alpha=0.8$. The order $q$ of the structure function is $1 / 4$. Solid line indicates the relation $s=0.8$. The exponent $s$ for the second order structure function is indicated by crosses. Dotted line shows the "pseudo - Gaussian" relation $s=\nu+1 / 2$.

Fig. 5. (a) Exponent $H_{\nu}$ for the range, see Eqs. (19), (20), versus $\nu$ is depicted by black points. The numerical results are obtained with the use of the modified Hurst method proposed in the paper. Processes along the vertical dotted line $a$ from Fig.1 are investigated. The Levy index $\alpha$ is 1.7. Solid line indicates the relation $H_{\nu}=\nu+1 / \alpha$. (b) Exponent $H_{\nu}$ is indicated for the processes along the dotted line $b$ from Fig.1 (black points). The parameters of these processes obey the law $\nu+1 / \alpha=0.8$. The modified Hurst method is used. Solid line indicates the relation $H_{\nu}=0.8$. The exponent $H_{\nu}$, obtained with the use of "traditional" Hurst method, is indicated by crosses. Dotted line shows the "pseudo - Gaussian" relation $s=\nu+1 / 2$. 


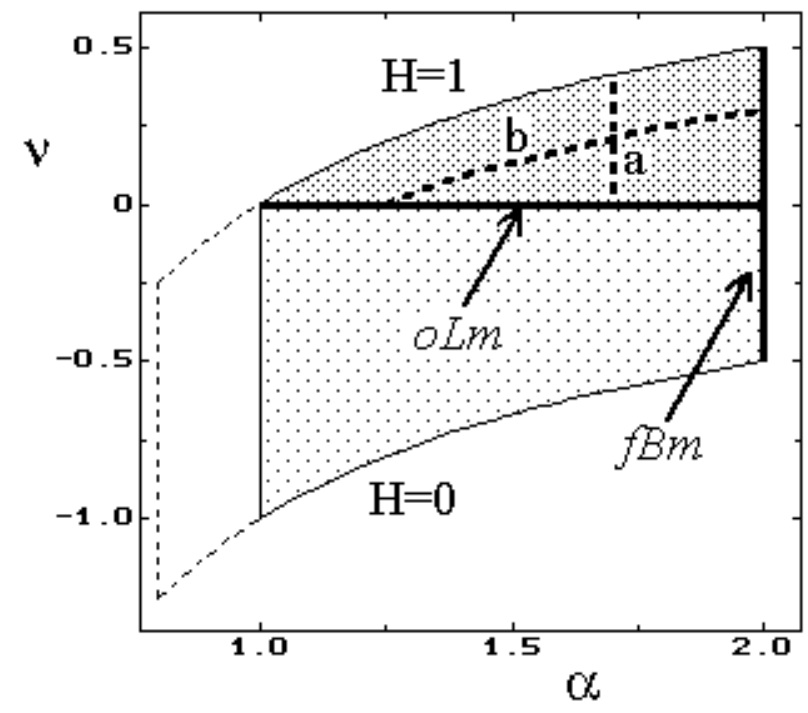



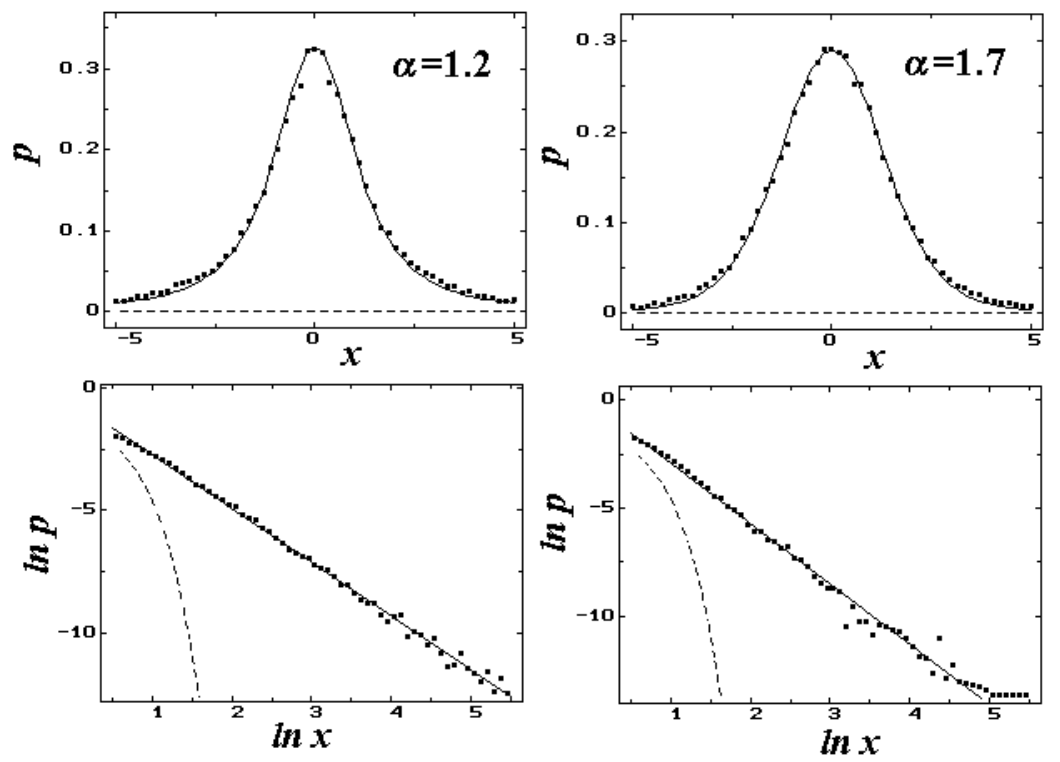

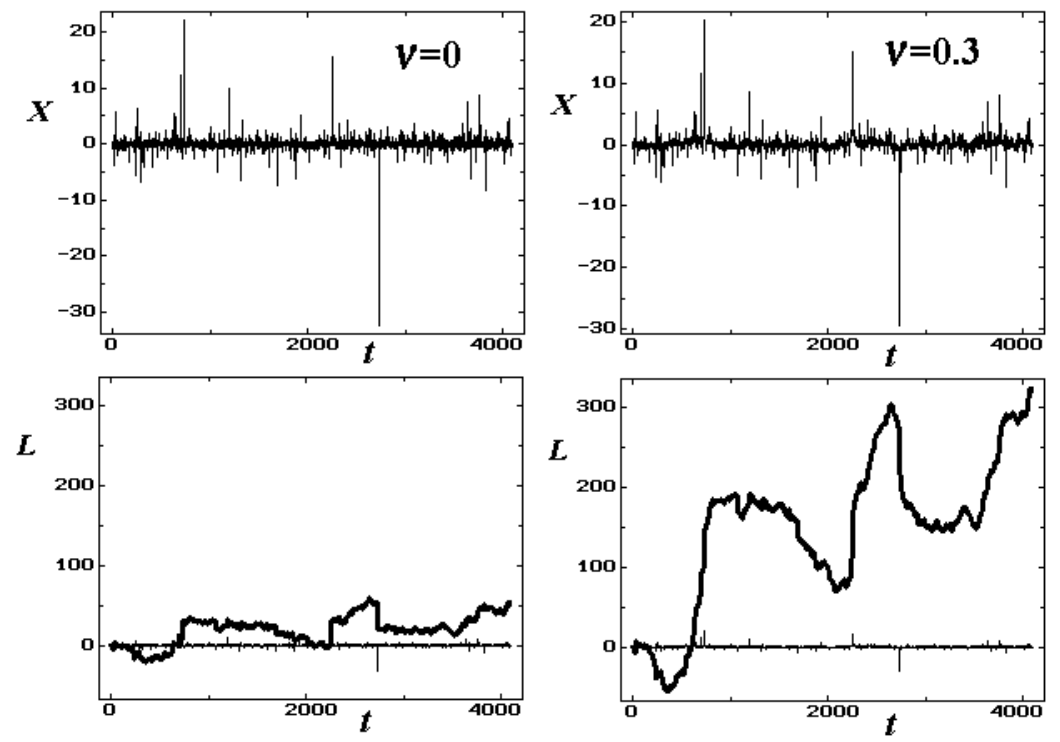

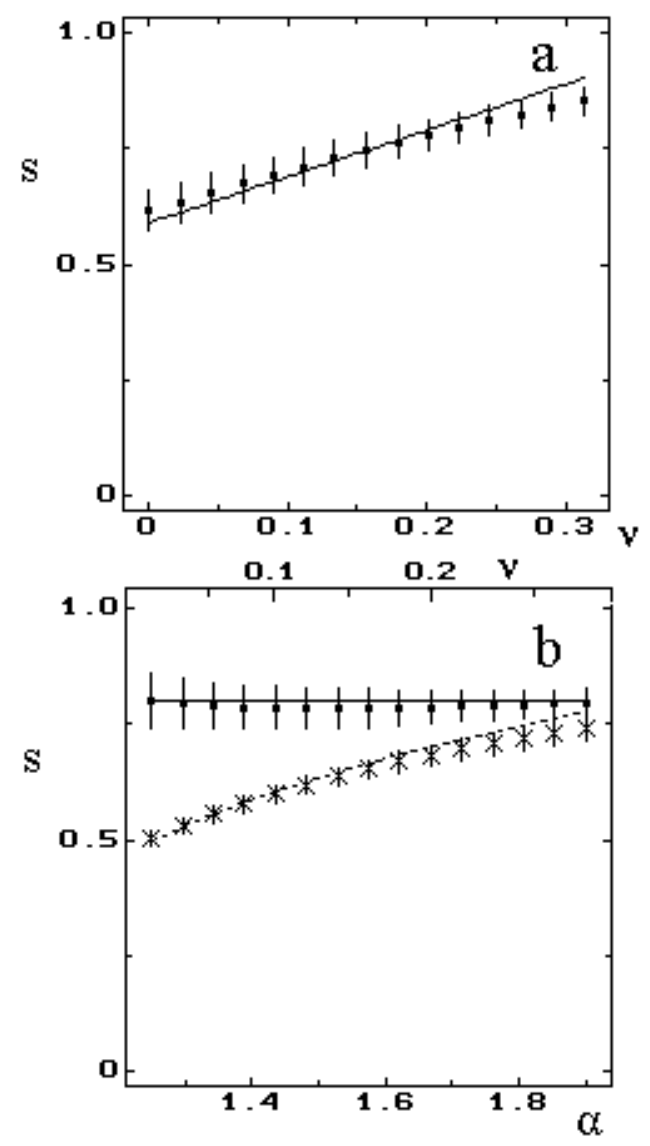

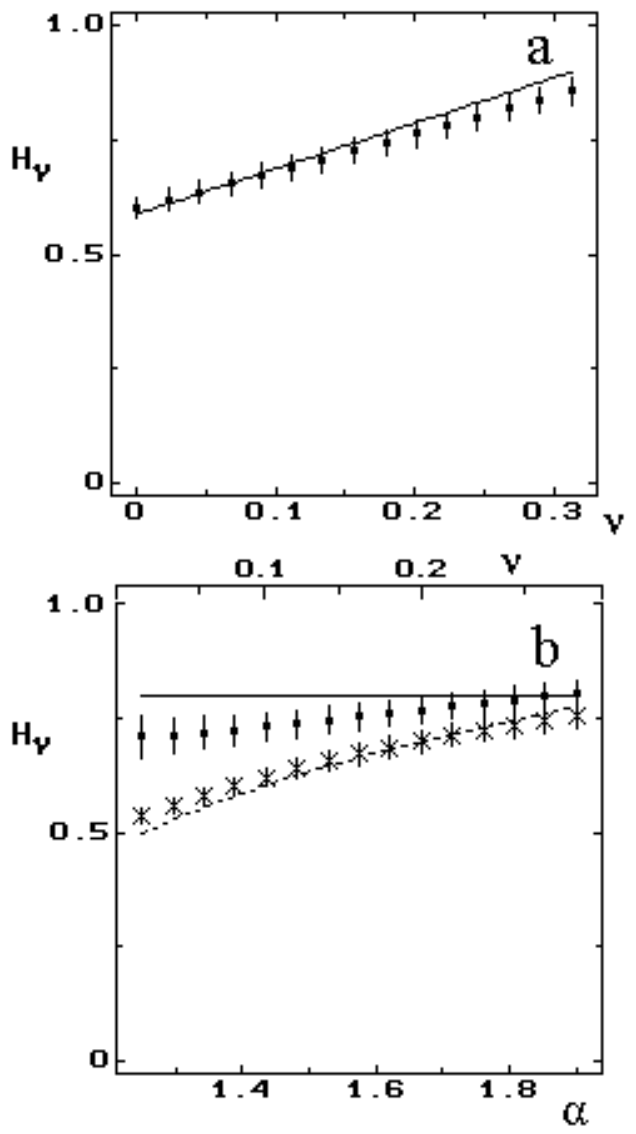